\title{
Influence of Emotional Burnout on Coping Behavior in Pedagogical Activity
}

\author{
Elena Voitenko $^{1 *}$, Serhii Myronets ${ }^{2}$, Vasyll Osodlo ${ }^{3}$, Kateryna Kushnirenko ${ }^{4}$, Ruslan \\ Kalenychenko ${ }^{5}$ \\ 1, 2,3, ${ }^{4}$ Department of Psychology, Kyiv National University of Trade and Economics, Ukraine \\ ${ }^{5}$ Department of Professional Education, Kyiv National University of Construction and Architecture, Ukraine
}

Keywords:

Professional stress, Emotional burnout, Coping behavior, Coping strategy

Received

3 April 2021

Received in revised form

25 April 2021

Accepted

27 April 2021

*Correspondence:

evoytenko73@gmail.com

\begin{abstract}
Emotional burnout syndrome is seen as a non-constructive mechanism of psychological protection, which worsens the psycho-emotional state and complicates professional and social adaptation. The study aims to identify the presence and nature of the relationship between the development of this syndrome in teachers with the degree of intensity of coping mechanisms. In a study organized by the cross-sectional design, a survey of 107 Ukrainian teachers was conducted. According to the purpose of the study, the following diagnostic techniques were used: Victor Boyko's method of diagnosing the level of emotional burnout, version of the Russian-language adaptation of Ways of Coping Questionnaire. The main hypotheses of this study were tested using correlation analysis and analysis of variance. Correlations between the stages of emotional burnout and the peculiarities of coping behavior have been established. It was found that the development of emotional burnout in teachers is associated with the gradual replacement of constructive coping strategies with non-constructive strategies. Furthermore, teachers' emotional burnout was associated with the gradual replacement of problem-oriented coping strategies with passive maladaptive strategies. Burnout due to the violation of the system of self-regulation and depletion of resources of stress resistance was accompanied by increased strategies of avoidance and emotional distancing from problems and strategies that involved a high degree of hostility and aggression. The obtained results indicated the inexpediency of considering coping strategies as a factor in overcoming emotional burnout. Further research is needed to study the factors and mechanisms for choosing coping strategies, criteria for their effectiveness, and consequences for further development of stress
\end{abstract}

CCIKD Publishing

Emotional burnout is a complex psychological phenomenon that often accompanies activities that require long-term involvement in continuous, direct contact with people in an emotionally saturated atmosphere. Some aspects of job outcomes are an output of the intercorrelated variables such as self-efficacy and psychological empowerment (Ardabili, 2020). Emotional burnout syndrome in professional activities is traditionally seen as a consequence of prolonged professional stress, which 
manifests itself as a reduced emotional background, indifference, a tendency to negative selfesteem, devaluation of their professional achievements (Maslach, 2003). The development of the syndrome leads to an increase in the number of conflicts with communication partners, degrades the quality of services provided, and creates dissatisfaction of employees with the results of their work, causes a loss of sense of life value and faith in their strength (Vodopyanova \& Starchenkova, 2008). In this regard, it is important to study the mechanisms of coping with stress in conditions of professional activity. Coping behavior is a conscious way of overcoming stress that mediates the relationship between a stressful event and a stress response and determines the degree of adaptation of the individual to the situation (Lazarus, 2006). It has been shown that insufficient adaptation to stress is associated with the lack of a flexible system of various coping strategies adequate to the situation's requirements and capabilities. The tendency to act in tense situations in an unconstructive manner leads to a chronic course of stress and burnout (Fomina \& Shabanova, 2015). The existence of conflicting data on the relationship between emotional burnout as a result of stress and coping strategies, which, on the one hand, are considered stabilizing factors, and on the other hand, lead to the development of mental maladaptation, shows the lack of theoretical basis. Factors and mechanisms for choosing coping strategies, as well as their effectiveness, remain insufficiently studied. In this regard, it is advisable to juxtapose the severity of emotional burnout with the degree of intensity of coping mechanisms in the behavior of teachers. The study of the relationship between coping strategies and the dynamics of emotional burnout of professionals can help solve the problem of its prevention and be used in teaching constructive behavior to overcome stressful situations. Thus, the main purpose of this study is to determine the presence and nature of the relationship between emotional burnout as a result of occupational stress with the peculiarities of coping behavior of teachers.

\section{Literature Review}

The study of various ways and mechanisms of human adaptation to the requirements of professional activity, which is directly related to the issue of stress, remains a relevant area of modern research. It is shown that the syndrome of emotional burnout is more often observed among the representatives of the communicative professions, which are associated with intensive interpersonal contacts (Ronginskaya, (2002). The main role in developing burnout belongs to emotionally complex or tense relationships at the "man-man" system (Vodopyanova \& Starchenkova, 2008). One of the most emotionally stressful types of professional activity is the work of a teacher. Pedagogical activity is constantly associated with intensive communication, purposeful perception of partners, and influence on them. The work of a teacher requires high qualification, associated with the need to meet the high level of social expectations of others, to acquire new knowledge constantly, has a high degree of responsibility for the results of work (Korytova, 2005). Difficulties due to the specifics of pedagogical activities lead to the emotional devastation of professionals, reducing the level of their professional suitability. The consequences of burnout are inevitably manifested in professional mistakes and conflicts and emotional breakdowns, in disorders of social adaptation, in the development of various psychosomatic, neurotic, stress-related, somatoform disorders (Maslach, 1982).

Coping behavior is emphasized as a major variable that affects the ability to withstand the negative effects of occupational stressors (Lazarus \& Folkman, 1984). Different individuals react differently to the same stressor. The type of response and direction of behavior depends on the 
person's individual characteristics and, particularly, on his individual psychological resources of overcoming (Voitenko, 2020).

The question of effective and ineffective coping behavior is directly related to the concept of coping strategies. The concept of "coping strategy" means the techniques and methods by which the process of overcoming stress is carried out. Coping is characterized by approaching stressors (aggression, information retrieval, problem-solving) or avoidance of stressors (ignoring, distraction, distancing), which are manifested on the cognitive, emotional, and behavioral levels. These characteristics underlie various coping behavior strategies (Lazarus, 2006). Coping behavior includes all intentional attempts of a person to cope with stress, change the situation for the better, and not just successful efforts. The psychological purpose of coping is to adapt a person to the requirements of the situation, master it, weaken or mitigate the requirements, and neutralize the stress impact of the situation (Losoya, Eisenberg, \& Fabes, 1998).

There is currently no generally accepted classification of types of coping behavior. However, most often, researchers rely on the concept of two coping behavior strategies proposed by Lazarus and Folkman (1984): problem-oriented and emotion-focused coping behavior. The first type of coping is associated with the mobilization of behavior resources and is manifested in the rational analysis of the problem, in seeking help from other people. Coping focused on emotions is manifested in the mobilization of resources to regulate emotional experiences. Efforts to regulate emotional experiences can take various forms: distraction from negative emotions, distancing from the problem, focusing on the positive aspects of the situation (Korytova, 2005; Varyihanova et al., 2012).

Coping behavior is triggered by a specific situation, which sets its features - "situational factor" (Kryukova, 2004). In particular, assessing the degree of the stressfulness of the situation affects the choice of coping strategies. The effectiveness of a strategy depends on the characteristics of the current situation and available personal resources, so we can talk about individual coping strategies' situational adaptability or maladaptability. Strategies that are effective in one situation can be ineffective and even harmful in another.

The most adaptive coping strategies aim directly at solving the problem situation (Carver et al., 1989). The authors included the following coping strategies: 1) "active coping" - active actions to eliminate the source of stress, 2) "planning" - planning their actions in relation to the problem situation, 3) "search for active public support" - seeking help, advice from their social environment, 4) "positive interpretation and growth" - assessment of the situation in terms of its positive aspects and attitude to it as one of the episodes of their life experience, and 5) "acceptance" - recognition of the reality of the situation. Another block of coping strategies, according to the authors we studied, may also contribute to adaptation in a stressful situation, but it is not associated with active coping. Such strategies of coping behavior include: 1) "search for emotional social support" - the search for compassion and understanding from others, 2) "suppression of competitive activity" - a decrease in activity on other matters and problems and a full focus on sources of stress, and 3) "containment" - the expectation of more favorable conditions for resolving the situation. The third group of coping strategies is not adaptive, but in some cases, it helps to adapt to a stressful situation and cope with it. These included: 1) "focus on emotions and their expression" - emotional response in a problem situation, 2) "denial" - denial of a stressful event, 3) "mental alienation" psychological distraction from the source of stress through entertainment, dreams, sleeping, etc., and 4) "behavioral exclusion" - a refusal to resolve the situation. 
Under the influence of living conditions and professional activities, in response to environmental stressors, builds their style of coping behavior (Isaeva, 2009). The study of teachers' coping behavior structure showed a clear dominance of a specific coping strategy (Korytova, 2005). It means that a tense situation encourages them to the usual stereotypes of behavior. Lack of a flexible system of coping strategies for teachers, adequate to the requirements of the situation and their capabilities, reduces the effectiveness of adaptation to occupational stress, leads to its chronic course and emotional burnout (Fomina \& Shabanova, 2015). Some studies have shown that constructive coping strategies help overcome burnout, while passive coping strategies, on the contrary, enhance the process of its formation (Varyihanova et al., 2012). The presence in the scientific literature of contradictory data on the relationship of coping behavior, stress dynamics, and burnout shows the lack of theoretical basis. Some authors note that coping behavior can help overcome stress, reduce or mitigate the impact of adverse factors, but can be unconstructive and ineffective, leading

Thus, theoretical analysis has shown that emotional burnout is a maladaptive non-constructive mechanism of psychological protection, which arises from insufficiently effective adaptation to long-term stress due to the lack of a flexible system of various coping strategies. Despite the fact that coping behavior aims to increase human adaptation to the environment, this process can be unconstructive and inefficient and lead to maladaptation. The identified contradictions suggest that the emotional burnout of teachers is interrelated with unproductive coping strategies. The development of emotional burnout worsens the psycho-emotional state. It complicates further professional and social adaptation connected with the gradual violation of self-regulation, depletion of individual resources of stress tolerance.

Thus, based on the results of theoretical analysis, we can formulate the general (theoretical) hypothesis of our study as follows: syndrome of emotional burnout has a negative impact on the repertoire of coping strategies in the behavior of teachers. The research program was based on an experimental hypothesis: the development of emotional burnout syndrome gradually narrows the range of coping strategies in the behavior of teachers, replacing constructive coping strategies with non-constructive strategies. Proving the experimental hypothesis requires answers to the following questions:

1. What are the features of the relationship between coping behavior and emotional burnout resulting from occupational stress?

2. How does the repertoire of coping strategies change with increasing levels of emotional burnout?

To answer these questions, two pairs of statistical hypotheses were formulated. The null and alternative hypotheses were formulated to statistically confirm the relationship between the characteristics of coping behavior and emotional burnout due to occupational stress.

$H_{0}$ : Differences between coping indicators are absent in groups of teachers with different levels of emotional burnout.

H1: There are differences between coping indicators in groups of teachers with different levels of emotional burnout. 
To statistically confirm the relationship between the development of symptoms of emotional burnout and the formation of coping strategies, two hypotheses were formulated:

Ho: Indicators of the level of prevalence of certain coping strategies do not correlate with the phases of emotional burnout (tension, resistance, exhaustion).

H1: Indicators of the level of prevalence of certain coping strategies correlate with the phases of emotional burnout (tension, resistance, exhaustion).

\section{Method}

A study to determine the nature of the relationship between coping rates and emotional burnout as a result of occupational stress in pedagogical aktivity was organized by using a cross-sectional method. The participants of the study were Ukrainian teachers of higher educational institutions, selected by random sampling. The accessible sample was 110 subjects. The questionnaires of three respondents were removed due to incomplete answers. Questionnaires of 107 respondents were analyzed. This sample size is sufficient for a study to identify the main trends in the relationship between the selected variables (Nasledov, 2004). The socio-demographic characteristics of respondents are presented in Table 1.

Table 1

Demographic Characteristics of Respondents

\begin{tabular}{lcc}
\hline & & Percentage $\%$ \\
\hline Gender & Male & 30.8 \\
& Female & 69.2 \\
Age & $25-35 \mathrm{yrs}$ & 18.7 \\
& $36-45 \mathrm{yrs}$ & 27.1 \\
& $46-55 \mathrm{yrs}$ & 46.7 \\
& $>56 \mathrm{yrs}$ & 7.5 \\
Experience & $1-10 \mathrm{yrs}$ & 24.3 \\
& $11-20 \mathrm{yrs}$ & 30.8 \\
& $21-30 \mathrm{yrs}$ & 34.6 \\
& $>30 \mathrm{yrs}$ & 10.3 \\
\hline
\end{tabular}

\section{Materials}

The following diagnostic tools were used: the method of diagnosing the level of emotional burnout Victor Boyko (Boyko, 2008), the version of the Russian-language adaptation of the Ways of Coping Questionnaire (WCQ) developed by Lazarus and Folkman (Bityutskaya, 2015).

The questionnaire "Diagnosis of the level of emotional burnout", developed and standardized by Boyko (2008), was used to identify the level of development of emotional burnout and its individual components. The choice of the questionnaire was based on the fact that it is designed to assess burnout associated with work stress and is known as a reliable and valid diagnostic tool (Raygorodsky, 2001). The tool allows identifying the main symptoms of emotional burnout, which represent three phases of its formation. The tension phase is the experience of traumatic circumstances, self-dissatisfaction, anxiety, and depression. The resistance phase is an inadequate selective emotional reaction, emotional and moral disorientation, expansion of the sphere of preservation of emotions, reduction of professional responsibilities. The exhaustion phase is emotional deficit and alienation, depersonalization, psychosomatic and psychovegetative disorders. The method is to fill out a questionnaire of 84 items; each question provides a clear answer, "yes" or "no". The questions are grouped into three scales, which allows diagnosis of the formation of 
each phase. Each scale is divided into four subscales, representing certain symptoms: 36 points or less - the phase is not formed; 37-60 points - the phase is developing; 61 points or more - the phase has developed.

The Ways of Coping Questionnaire (WCQ), developed by Lazarus and Folkman (1984), was used to diagnose coping strategies. The Russian-language adaptation of the questionnaire was contextualized and standardized by Bityutskaya at Lomonosov Moscow State University (Bityutskaya, 2015). This questionnaire is also considered a valid and reliable diagnostic tool (Lugovskaya \& Assanovich, 2017). The questionnaire was specifically designed to measure coping with stress in specific situations. It consists of eight subscales, including coping techniques such as confrontation, distancing, self-control, seeking social support, taking responsibility, avoiding, solving problems, and positively reassessing the situation. The processing of the results is the summation of points for each scale and then their calculation according to the formula: $\mathrm{X}=$ sum of points/max score $* 100$. The questionnaire offers 50 items and four answer options: never - 0 points; rarely - 1 point; sometimes - 2 points; often - 3 points.

\section{Analysis}

The main hypotheses of this study were tested using correlation analysis and analysis of variance. Data processing was performed using IBM SPSS Statistics 22. Acceptance or rejection of statistical hypotheses formulated to confirm the relationship between the characteristics of coping behavior and emotional burnout due to occupational stress was carried out using the procedure of analysis of variance. The correctness of the application of analysis of variance to the scales of all selected questionnaires was checked using the Homogeneity of variance test Levene. The Brown-Forsythe criterion for the validity of conclusions about differences between means was applied in the case of discrepancy. The Tukey post hoc test and the Games-Howell test were used to determine the characteristics of the influence.

Acceptance or rejection of statistical hypotheses formulated to confirm the relationship between the manifestation of symptoms of emotional burnout and the formation of coping strategies was carried out using correlation analysis. The correctness of the application of Spearman correlation analysis is conditioned by the affiliation of all scales of the selected methods to the ordinal and the inconsistency of the obtained frequency distribution values to the normal distribution.

\section{Results}

The results of the analysis of variance are presented in Table 2. According to the results of the analysis of variance, it was determined that the stage of Tension did not affect the results only on three scales of the coping test, namely: Self-control, Avoidance, and Positive reassessment ( $p$ > .05). The Resistance stage did not affect the results on only two scales of the coping test, namely: Search for social support and Planning a solution to the problem $(p>.05)$. The stage of Exhaustion did not affect the results on only two scales, namely: Self-control and Positive reassessment ( $p>$ .05). Additional application of the Brown-Forsythe criterion denied the legitimacy of the conclusions about the differences between the averages on the scale of coping test "Planning a solution to the problem" ( $p>.05)$. Therefore, the data on this scale were excluded from further analysis. Indicators on other scales of WCQ were significantly influenced by the stages of emotional burnout of teachers (Table 2). 
Table 2

The Results of Analysis of Variance

\begin{tabular}{|c|c|c|c|c|c|c|c|c|c|}
\hline Burnout phases & & Tension & & & esistan & & & Exhaustic & \\
\hline Coping Strategies & $\mathrm{F}$ & MSE* & $\mathrm{p}$ & $\mathrm{F}$ & MSE* & $\mathrm{p}$ & $\mathrm{F}$ & MSE* & $\mathrm{p}$ \\
\hline Confrontational coping & 4.63 & 52.60 & $\leq .05$ & 4.04 & 52.60 & $\leq .05$ & 4.64 & 52.60 & $\leq .05$ \\
\hline Distancing & 5.14 & 55.50 & $\leq .01$ & 3.07 & 55.50 & $\leq .05$ & 5.19 & 55.50 & $\leq .01$ \\
\hline Self-control & - & - & - & 5.31 & 53.40 & $\leq .01$ & - & - & - \\
\hline Search for social support & 3.92 & 51.92 & $\leq .05$ & - & - & - & 14.78 & 51.92 & $\leq .001$ \\
\hline Acceptance of responsibility & 3.44 & 50.04 & $\leq .05$ & 3.93 & 50.04 & $\leq .05$ & 3.31 & 50.04 & $\leq .05$ \\
\hline Avoidance & - & - & - & 3.57 & 52.56 & $\leq .05$ & 10.59 & 52.56 & $\leq .001$ \\
\hline $\begin{array}{l}\text { Planning a solution to the } \\
\text { problem }\end{array}$ & 3.75 & 55.64 & $\leq .05$ & - & - & - & - & - & - \\
\hline Positive revaluation & - & - & - & 3.75 & 54.70 & $\leq .05$ & - & - & - \\
\hline
\end{tabular}

The Tukey post hoc test and the Games-Howell test were used to determine the characteristics of the influence. Analysis of the nature of the links, proven by significant differences between the indicators of coping in groups of teachers with low (1), medium (2), high (3) levels of emotional burnout phases "Tension", "Resistance", "Exhaustion", allows us to identify two trends, which confirm the statistical hypothesis, but have different characteristics of the differences.

First, in groups of teachers with different levels of emotional burnout, significant linear differences between indicators of coping behavior were found, which proves an alternative statistical hypothesis. The linear nature of these differences suggests that an increase in tension level leads to an intensification of coping behavior. This trend is confirmed by the distribution of coping indicators in groups of teachers with low (1), medium (2), and high (3) levels of the phase "Tension" on the following scales (Figure 1): "Confrontational coping" (significant differences were found between the low and medium levels $(* * p \leq .01)$ (Figure 1a); "Distancing" (significant differences were found between the low and medium levels $(* * p \leq .01)$ (Figure 1b). On the scale of "Acceptance of responsibility," no significant differences were found between the levels.

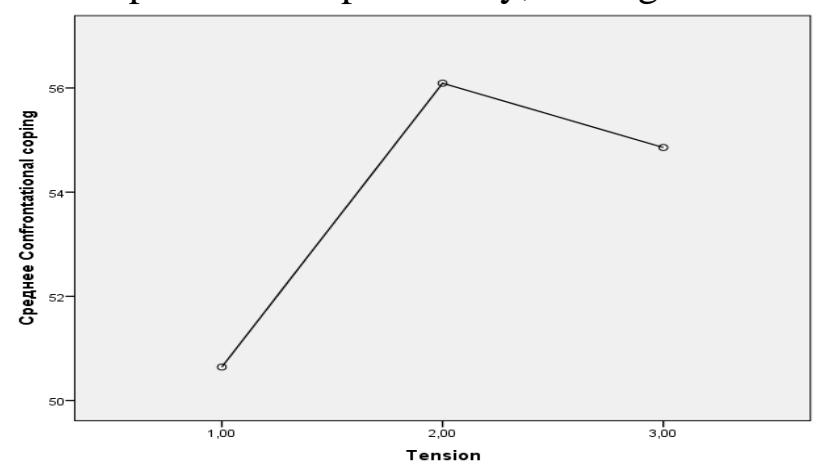

a)

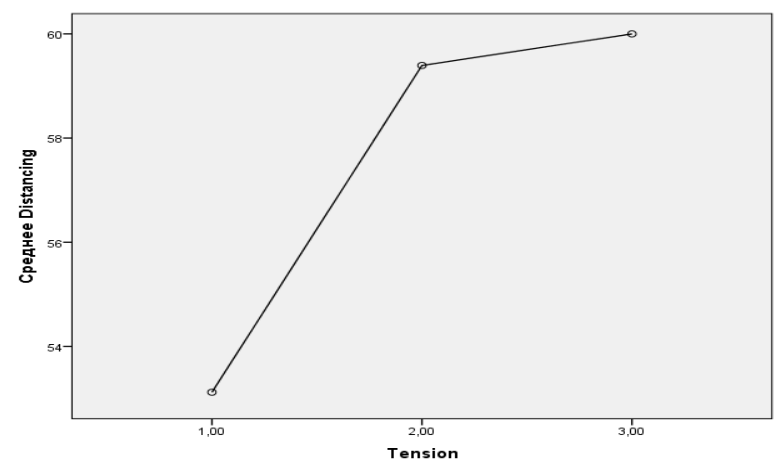

b)

Figure 1. Linear differences between coping indicators in groups of teachers with low (1), medium (2), and high (3) levels of the phase of emotional burnout "Tension"

Second, significant nonlinear differences were found between coping indicators in groups of teachers with different levels of emotional burnout, which also confirms an alternative statistical hypothesis about the existence of such differences (Figure 2). Dynamics of change of average values of coping behavior on scales "Search of social support" and "Planning of the decision of a problem" in groups of teachers with low (1), medium (2), and high (3) levels of a phase of emotional burnout "Tension" has no expressed linear character: "Search for social support", significant differences were found between medium and high levels $\left({ }^{*} p \leq .05\right)$ (Figure $\left.2 \mathrm{a}\right)$; "Planning a solution 
to the problem", significant differences were found between low and medium levels $(* * p \leq .01)$ and also between medium and high levels $(* * p \leq .01)$ (Figure $2 \mathrm{~b}$ ), and at a high level.

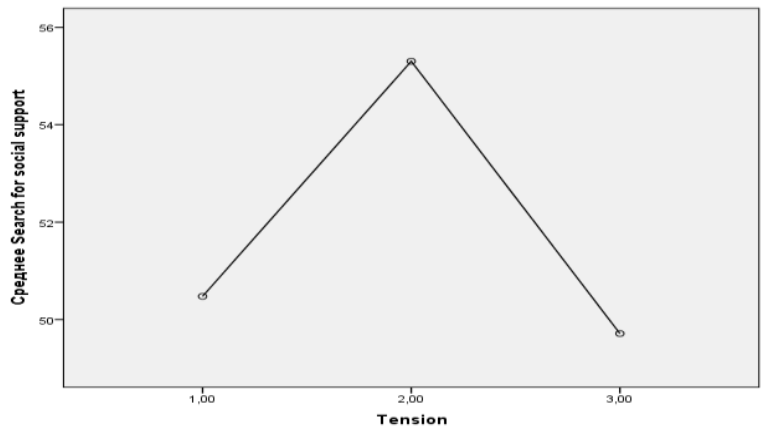

a)

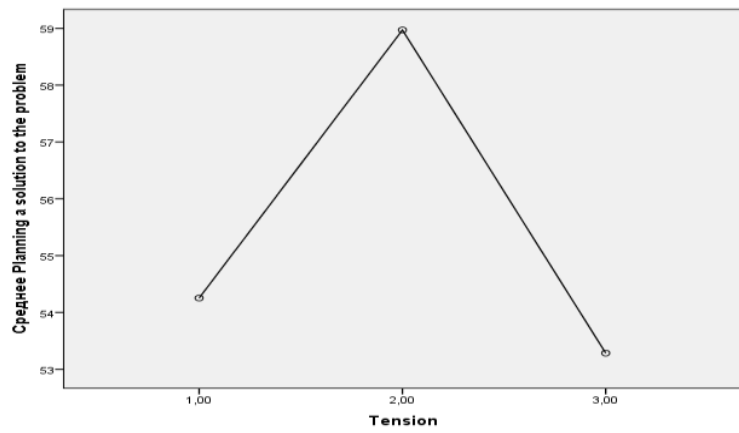

b)

Figure 2. Nonlinear differences between coping indicators in groups of teachers with low (1), medium (2), and high (3) levels of emotional burnout phase "Tension"

Differences between coping indicators in groups of teachers with low (1), medium (2), and high (3) levels of the phase of emotional burnout "Resistance" also have different characteristics of manifestation. Coping indicators are linear in groups of teachers with low (1), medium (2), and high (3) levels of the phase of emotional burnout "Resistance" on the following scales (Figure 3): "Self-control", significant differences were found between low and high levels $\left({ }^{*} p \leq .05\right)$ and also between medium from high levels $(* p \leq .05)$ (Figure 3a); "Acceptance of responsibility", significant differences were found between low and medium levels $\left({ }^{*} p \leq .05\right)$ and also between low and high levels $(* p \leq .05)$ (Figure 3b), "Avoidance", significant differences were found between low and high levels $(* p \leq .05)$ (Figure $3 \mathrm{c}$ ). According to the "Distancing" scale, no significant differences between the indicators of coping at the three levels of the phase of emotional burnout "Resistance" were found.

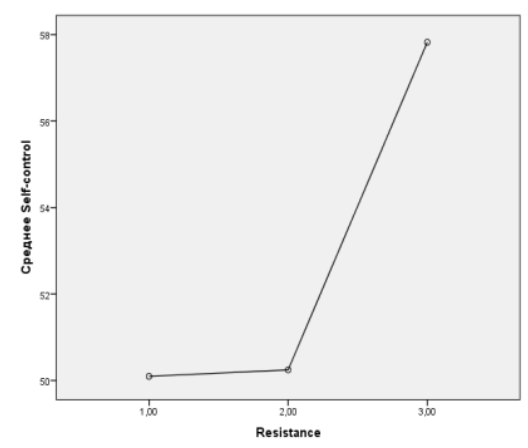

a)

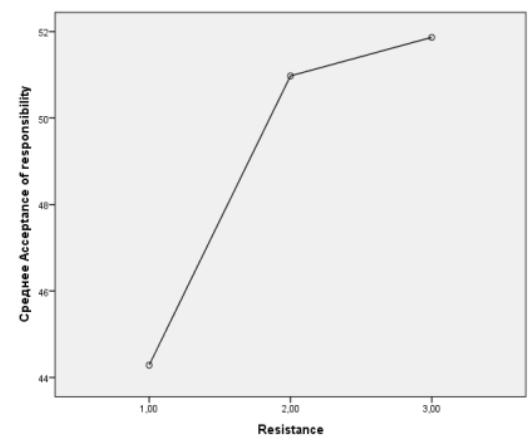

b)

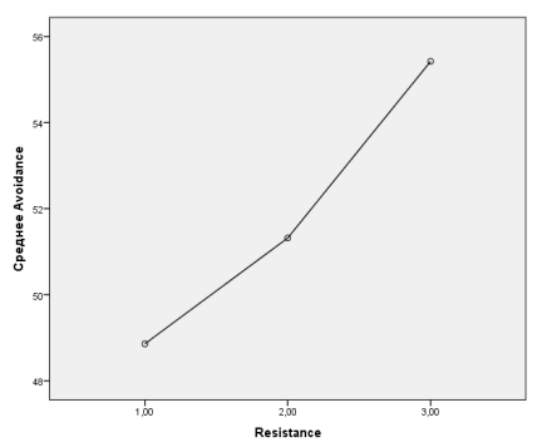

c)

Figure 3. Linear differences between coping indicators in groups of teachers with low (1), medium (2), and high (3) levels of emotional burnout phase "Resistance"

The distribution of coping indicators in groups of teachers with low (1), medium (2), and high (3) levels of emotional burnout phase "Resistance" on the scales "Confrontational coping" and " Positive revaluation" is nonlinear (Figure 4). Dynamics of change of average values of coping behavior on scales "Confrontational coping" and "Positive revaluation" in groups of teachers with low (1), average (2) and high (3) levels of a phase of emotional burnout "Resistance" don`t have expressed linear character: "Confrontational coping", significant differences were found between low and medium levels $(* p \leq .05)$ (Figure $4 \mathrm{a}$ ); "Positive revaluation", significant differences were 
found between medium and high levels of the resistance phase $\left({ }^{*} p \leq .05\right)$, and, at a high level, these figures are lower (Figure 4b).

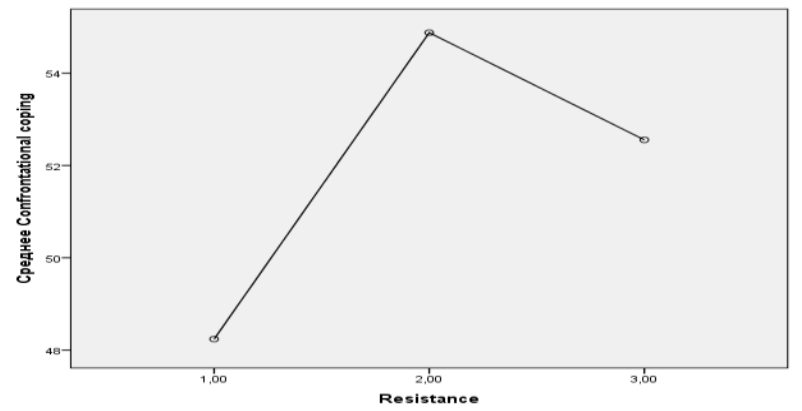

a)

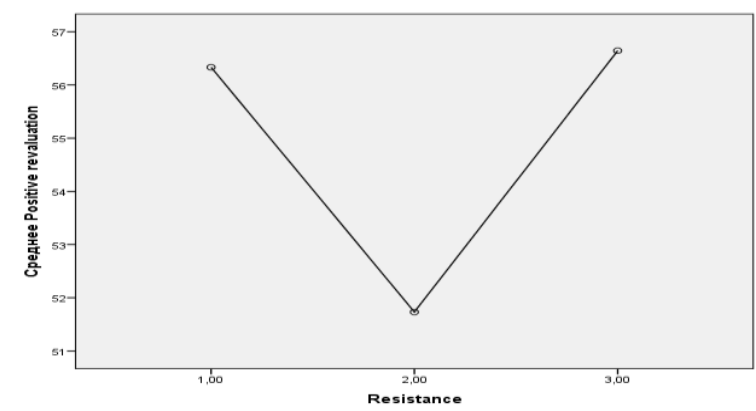

b)

Figure 4. Nonlinear differences between coping indicators in groups of teachers with low (1), medium (2), and high (3) levels of emotional burnout phase "Resistance"

The differences between the indicators of coping in the groups of teachers with low (1), medium (2), and high (3) levels of the phase of emotional burnout "Exhaustion" also have ambiguous characteristics. The distribution of indicators of coping behavior is linear in groups of teachers with low (1), medium (2), and high (3) levels of manifestation of the phase of emotional burnout "Exhaustion" on scales (Figure 5): "Distancing", significant differences were found between low and medium levels $(* * p \leq .01)$ and also between low and high levels (**p $\leq .01)$ (Figure 5a); "Search for social support", significant differences were found between low and medium levels $(* * * p \leq .001)$ and also between low and high levels $(* * * p \leq .001)$ (Figure $5 \mathrm{~b}$ ), "Acceptance of responsibility", significant differences were found between low and high levels $(* * * p \leq .001)$ and also between medium and high levels $(* * p \leq .01)$ (Figure $5 \mathrm{c}$ ).

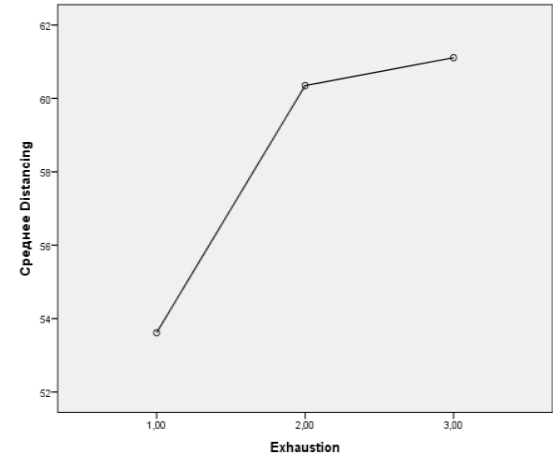

a)

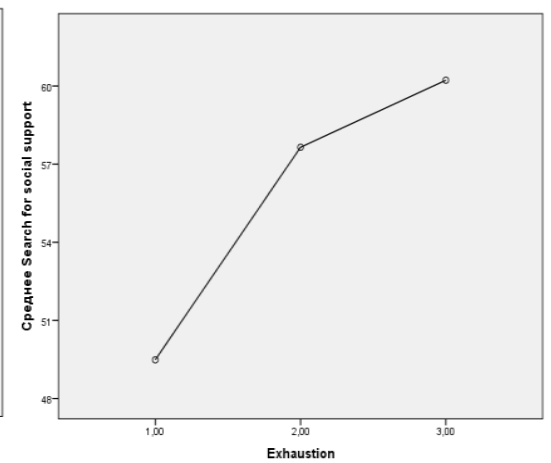

b)

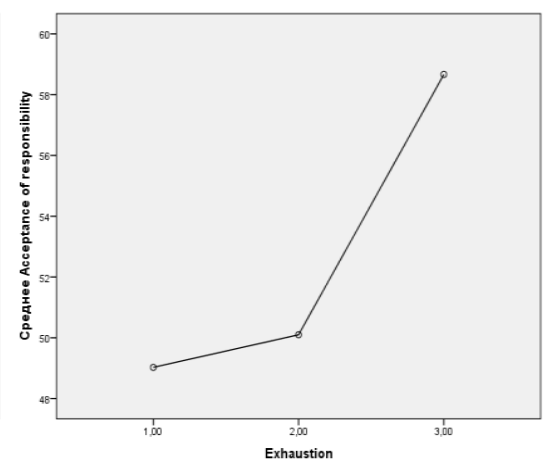

c)

Figure 5. Differences between coping indicators in groups of teachers with low (1), medium (2), and high (3) levels of emotional burnout phase "Exhaustion"

The distribution of average values of coping indicators on the scales "Avoidance" and "Confrontational coping" in teachers with low (1), medium (2), and high (3) levels of the phase of emotional burnout "Exhaustion" is nonlinear (Figure 6.). The average values of coping indicators at different levels of the "Exhaustion" phase showed that the gradual growth is not monitored on the scale "Confrontational coping", because the average indicators are the highest at the average level of coping. However, significant differences were found only between low and medium levels $\left(*^{*} p \leq .01\right.$ ) (Figure 6a). Therefore, it can be assumed that the indicators for this coping strategy were also increased according to the levels of "Exhaustion". The second trend is also confirmed by the distribution of average values of coping indicators on the scale "Avoidance" in teachers with 
low (1), medium (2), and high (3) levels of the phase of emotional burnout "Exhaustion" (Figure $6 b)$.

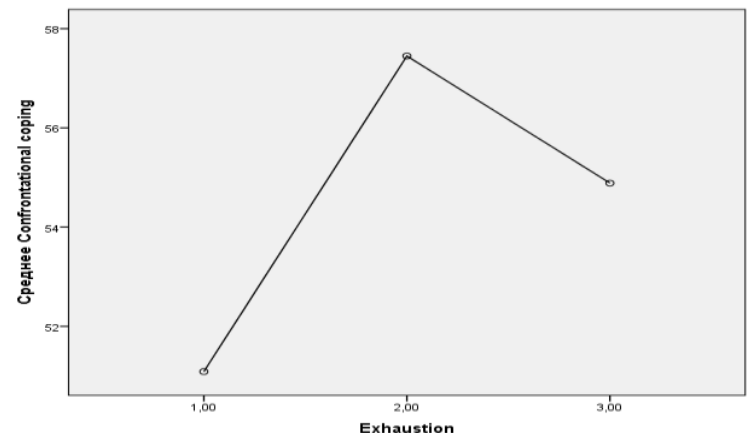

a)

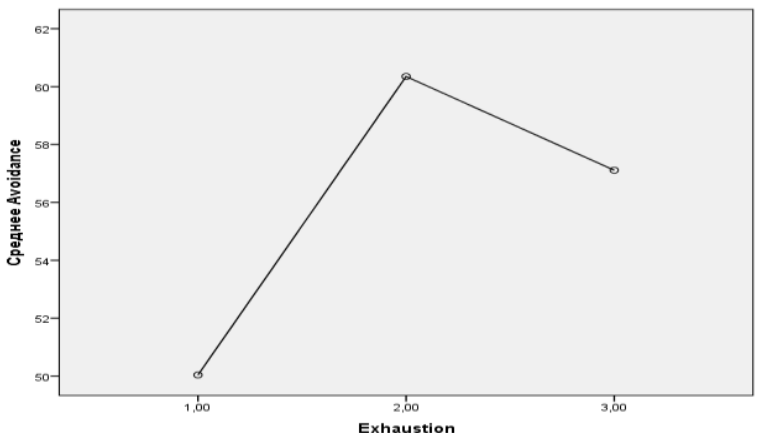

b)

Figure 6. Nonlinear differences between coping indicators in groups of teachers with low (1), medium (2), and high (3) levels of the phase of emotional burnout "Exhaustion"

Tukey's criterion divides levels into two subsets: the first combines low and high levels, the second combines high and medium. That is, a high level is an intermediate between low and medium levels of Exhaustion. The observed trend shows that both inflated and reduced indicators of the coping strategy "Avoidance" are associated with the development of the Exhaustion phase.

Correlation analysis revealed significant positive relationships between the phases of emotional burnout (Tension, Resistance, Exhaustion) and the level of preference for certain coping strategies (Table 3).

The presence of linearly significant positive connections between the Tension phase in the process of emotional burnout and coping strategies was revealed: Confrontational coping $(r=.4, p$ $\leq .01)$; Distancing $(r=.3, p \leq .01)$; Self-control $(r=.2, p \leq .05)$; Search for social support $(r=.3$, $p \leq .01)$; Acceptance of responsibility $(r=.47, p \leq .01)$; Avoidance $(r=.37, p \leq .01)$; Planning a solution to the problem $(r=.19, p \leq .05)$; Positive revaluation $(r=.2, p \leq .05)$.

The presence of linearly significant positive relationships between the phase of Resistance in the process of emotional burnout and certain coping strategies was revealed: Search for support ( $r$ $=.25, p \leq .01)$, Acceptance of responsibility $(r=.2, p \leq .05)$, Self-control $(r=.34, p \leq .01)$, Confrontational coping $(r=.2, p \leq .05)$, Distancing $(r=.2, p \leq .05)$, Avoidance $(r=.28, p \leq .01)$.

There are linearly significant positive relationships between the phase of Exhaustion in the process of emotional burnout and certain coping strategies was revealed: Confrontational coping ( $r$ $=.45, p \leq .01)$; Search for social support $(r=.36, p \leq .01)$; Acceptance of responsibility $(r=.2, p$ $\leq .05)$; Avoidance $(r=.4, p \leq .01)$.

Table 3

Correlation between Variables

\begin{tabular}{|c|c|c|c|c|}
\hline \multirow[t]{2}{*}{ № } & \multirow[t]{2}{*}{ Variables } & \multicolumn{3}{|c|}{ Emotional burnout phases } \\
\hline & & Tension & Resistance & Exhaustion \\
\hline 4 & Confrontational coping & $.4 * *$ & $.2 *$ & $.45^{* *}$ \\
\hline 5 & Distancing & $.3 * *$ & $.2 *$ & .18 \\
\hline 6 & Self-control & $.2 *$ & $.34 * *$ & .07 \\
\hline 7 & Search for social support & $.3 * *$ & $.25 * *$ & $.36 * *$ \\
\hline 8 & Acceptance of responsibility & $.47 * *$ & $.2 *$ & $.2 *$ \\
\hline 9 & Avoidance & $.37 * *$ & $.28 * *$ & $.4 * *$ \\
\hline 10 & Planning a solution to the problem & $.19^{*}$ & -.05 & .02 \\
\hline 11 & Positive revaluation & $.2 *$ & .1 & .02 \\
\hline
\end{tabular}




\section{Discussion}

This study aimed to identify the presence and nature of the relationship between the development of emotional burnout and the degree of intensity of coping mechanisms in teachers. Analysis of the nature of relationships, proven by significant differences between indicators of coping behavior in groups of teachers with low (1), medium (2), and high (3) levels of development of phases of emotional burnout "Tension", "Resistance", "Exhaustion", allowed to identify two trends that confirm the alternative statistical hypothesis, but have different characteristics of the differences.

First, in groups of teachers with different levels of emotional burnout, significant linear differences between indicators of coping behavior were found. The linear nature of these differences suggests that an increase in tension level leads to an intensification of coping behavior. It is shown that, since copings are a reaction to certain stressors, with the rise in the level of a certain phase of burnout, the indicators of copings also increase (Varykhanova et al., 2012). Analysis of variance, in general, confirms this obvious opinion but also highlights some deviations from it. Analysis of the average values of coping indicators at different levels of development of each phase of emotional burnout showed that the gradual growth is not constantly monitored. According to the indicators of coping strategies "Search for social support" and "Planning a solution to the problem" significant differences were recorded at the middle and a high level of the Tension phase, and at a high level these indicators are lower. We can conclude that the constructive coping strategies "Search for social support" and "Planning a solution to the problem" are not used by teachers when they have reached the highest level of the Tension phase. With the development of the phase of emotional burnout Resistance, a decrease in the level of application of the coping strategy "Positive Revaluation" has also been recorded. The differences between the coping strategy "Avoidance" indicators in groups of teachers with different levels of the phase of emotional burnout "Exhaustion" also have ambiguous characteristics. The observed trend shows that both inflated and decreased indicators of the coping strategy "Avoidance" are associated with the development of the Exhaustion phase. Our data contradict the results of studying coping behavior strategies as a factor in overcoming emotional burnout, which showed that constructive coping behavior strategies help to overcome emotional burnout, and passive coping strategies, on the contrary, enhance the process of its formation (Varykhanova et al., 2012). Our results show that with the formation of emotional burnout, constructive coping strategies are gradually being replaced by passive, maladaptive strategies to overcome stress, which, in turn, further worsens the psycho-emotional state of teachers, reducing professional and social adaptation.

The analysis of the identified correlations shows a gradual change in the repertoire of coping strategies in the behavior of the studied teachers in connection with the development of emotional burnout, which confirms the statistical hypothesis about the relationship between emotional burnout symptoms and the formation of coping strategies. This change is in the direction of gradually narrowing the range of coping strategies in the process of developing emotional burnout, as well as in the rejection of problem-oriented coping.

Correlation analysis showed that a wide range of possible coping strategies is presented at the stage of emotional burnout. Nervous (anxious) tension serves as a predictor and "trigger" mechanism in the formation of emotional burnout (Boyko, 2008). With increasing stress, the studied teachers tend to increase control over emotions and actions, recognize their role and responsibility in problems while striving to actively seek and devise ways to systematically overcome difficulties. 
Correlation analysis also showed that the stages of emotional burnout "Resistance" and "Exhaustion" do not correlate with Strategies for "Planning a Problem solution" and "Positive Revaluation", which are seen by researchers as adaptive, contributing to constructive problem solving (Carver et al., 1989; Lazarus \& Folkman, 1984). The predominance of emotionally oriented coping strategies in the behavior of respondents indicates a desire for psychological comfort, reducing the pressure of external circumstances through the exchange of feelings, thoughts, and actions aimed at meeting the need to improve the emotional state of a person under stress, maintaining relationships with others. However, these strategies are assessed as less effective than problem-oriented coping (Lasarus \& Folkman, 1984).

Psycho-emotional exhaustion, characterized by deformation of relationships with others, decreased overall energy tone, and weakening of the nervous system (Boyko, 2008; Maslach, 1982), is accompanied by increased coping behavior strategies of avoidance and emotionally distancing, as well as strategies that involve a high degree of hostility and aggression.

The stage of Exhaustion also correlates with the strategy of "Acceptance of responsibility", which involves recognizing the subject's role in the problem and responsibility for its solution, in some cases with an explicit component of self-criticism and self-blame. This strategy reflects the desire of the individual to understand the relationship between their actions and their consequences, the willingness to analyze their behavior, to look for the causes of current difficulties in personal shortcomings and mistakes. However, this strategy in behavior can lead to unwarranted selfcriticism, feelings of guilt, and dissatisfaction. These features are known to be a risk factor for depressive disorders (Boyko, 2008).

The Exhaustion stage is also accompanied by the abandonment of "Distancing" and "SelfControl" strategies. Distancing strategy involves attempts to overcome negative feelings about the problem by subjectively reducing its significance and the degree of emotional involvement in it (Boyko, 2008). The lack of an attempt to minimize the subjective significance of difficult situations is associated with the risk of intense emotional reactions to frustration. The strategy of "Selfcontrol" involves attempts to overcome negative feelings about the problem by purposefully suppressing and restraining emotions, minimizing their impact on assessing the situation and the choice of behavioral strategy, high control of behavior, the desire for self-control (Boyko, 2008). Accordingly, the probability of emotionally impulsive actions, loss of the rational approach to problem situations increases. The obtained results indicate the inexpediency of considering coping strategies as a factor in overcoming emotional burnout, contrary to some studies (Varyihanova et al., 2012).

\section{Conclusions and Future Study}

The study confirmed the assumption that emotional burnout has a negative impact on the repertoire of coping strategies in the behavior of teachers. It was found that certain symptoms of emotional burnout are present in most subjects. A close connection between the stages of emotional burnout and the peculiarities of coping behavior was identified. The study showed a gradual change in the repertoire of coping strategies in the behavior of the studied teachers in connection with the development of emotional burnout. This change is in the direction of gradually narrowing the range of coping strategies in the process of developing emotional burnout, as well as in the rejection of problem-oriented coping. 
It was also found that the development of emotional burnout in teachers is associated with the gradual replacement of constructive coping strategies with non-constructive strategies, which reduces the ability to adapt to the requirements of the situation and further exacerbates the psychological discomfort. Burnout due to a violation of the system of self-regulation and depletion of stress resources of the individual is accompanied by increased coping behavior strategies to avoid strong feelings and emotional distancing from problems and strategies that involve a high degree of hostility and aggression.

The obtained results indicate the inexpediency of considering coping behavior as a factor in overcoming emotional burnout. The psychological significance of coping is to more effectively adapt to the requirements of the situation, to weaken or mitigate these requirements, and thus reduce the effects of stress. With its inherent deformation of relationships with others, emotional exhaustion enhances the manifestation of psychological defense mechanisms. It encourages a person to the usual stereotypes of behavior, such as outbursts of irritability, aggression, quick exit from the situation (or refusal to solve). The unresolved situation, in turn, further exacerbates dissatisfaction with themselves and work and leads to the further development of burnout.

The results indicate the need for purposeful psychological assistance to teachers in managing professional stress and developing an effectively functioning system of self-regulation and coping behavior, taking into account the specifics of the activity, the typology of stressful situations, and individual factors.

Due to the limited sample, this conclusion is intermediate. Further research is needed to identify and classify different coping strategies caused by personal determinants, the specifics of professional activities, and types of stressful situations. The study of the relationship between coping strategies and the dynamics of stress can help solve the prevention of emotional burnout of professionals and be used in teaching constructive behavior to overcome stressful situations. It is also productive to identify the mediating role of certain coping strategies that significantly impact the success of professional adaptation in different organizational contexts.

\section{References}

Ardabili, F. (2020). Moderating-mediating effects of leader member exchange, self-efficacy and psychological empowerment on work outcomes among nurses. Organizacija, 53(3), 246-258. https://doi.org/10.2478/orga-2020-0016

Bityutskaya, E. V. (2015). Coping method questionnaire: a methodological guide. IIU MGOU.

Boyko, V. V. (2008). Psihoenergetika. Piter. Retreived from https://bookap.info/okolopsy/boyko_psihoenergetika/gl50.shtm

Carver, C. S., Scheier, M. F., \& Weintraub, J. K. (1989). Assessing coping strategies: a theoretically based approach. Journal of Personality and Social Psychology. 56, 267-283. Retrieved from https://pubmed.ncbi.nlm.nih.gov/2926629/

Fomina, N. V., \& Shabanova, T. L. (2015). Particular qualities of coping behavior among specialists in the social area: overcome resource or origin of chronic stress. Modern research of social problems, 7(51), 311-327.

Isaeva, E. R. (2009). Koping-povedenie i psihologicheskaya zaschita lichnosti v usloviyah zdorovya i bolezni [Coping behavior and psychological protection of a person in conditions of health and illness]. Izd-vo SPbGMU.

Korytova, G. S. (2005). Structure of defense and coping behavior in professional activity of pedagogical workers. Siberian Psychological Journal, 21, 112-117.

Kryukova, T. L. (2004). Psikhologiya sovladayushego povedeniya [The psychology of coping behavior]. Monografiya. KGU im. N.A. Nekrasova - «Avantitul».

Lazarus, $R$. (2006). Emotions and interpersonal relationships: Toward a person-centered conceptualization of emotions and coping. Journal of Personality, 74(1), 9-43. 
Lazarus, R., \& Folkman, S. (1984). Stress, appraisal and coping. Stress, appraisal, and coping. Springer Pub. Co.

Losoya, S., Eisenberg, N., \& Fabes, R. (1998). Developmental issues in the study of coping. International Journal of Behavioral Development, 22(2), 287-313.

Lugovskaya, A. A., \& Assanovich, M. A. (2017). Dyahnostycheskye svoistva modyfytsyrovannoi metodyky «Oprosnyk sposobov sovladanyia» R. Lazarusa [Diagnostic properties of the modified methodology "Ways of coping questionnaire" R. Lazarus]. Meditsinskie Novosti, 11, 61-65.

Maslach, C. (1982). Burnout: A social psychological analysis. In J. W. Jones (Ed.), The burnout syndrome: Current research, theory, interventions (pp. 30-53). London House.

Maslach, C. (2003). Job burnout: New directions in research and intervention. Current Directions in Psychological Science, 12, $189-192$.

Nasledov, A. D. (2004). Generalnaya sovokupnost i vyiborka [General population and sample] // Matematicheskie metodyi psihologicheskogo issledovaniya. Analiz i interpretatsiya dannyih. Uchebnoe posobie. 2-e izd., ispr. i dop. [In Mathematical methods of psychological research. Analysis and interpretation of data. Tutorial. 2nd ed., Rev. and add] (pp. 19-22). Izd. "Rech".

Raygorodsky, D. Ya. (Ed.). (2001). Prakticheskaya psihodiagnostika. Metodiki i testyi. Uchebnoe posobie. Red. [Practical psychodiagnostics. Methods and tests. Tutorial]. Izdatelskiy dom "BAHRAH-M". Samara, Rossiya: Bahrah-M.

Ronginskaya, T. I. (2002). Sindrom vyigoraniya v sotsialnyih professiyah [Burnout syndrome in social professions]. Psihologicheskiy Zhurnal, 23(3), 85-95.

Varyihanova, K. V., Linchuk, T. P., \& Chupina, E. A. (2012). Strategii sovladayuschego povedeniya kak faktor preodoleniya emotsionalnogo vyigoraniya u sotrudnikov pravoohranitelnyih organov [Coping Behavior Strategies as a Factor of Overcoming Emotional Burnout in Law Enforcement Officers]. Vestnik IrGTU, 8(67), 244-249.

Vodopyanova, N. E., \& Starchenkova E. S. (2008). Sindrom vyigoraniya: diagnostika i profilaktika [Burnout syndrome: diagnosis and prevention]. 2-e izd. Piter.

Voitenko, E. (2020). Emotional burnout as a result of professional stress in the work of managers. Behavior Studies in Organizations, 3, 5-12. https://doi.org/10.32038/JBSO.2020.03.02

Voitenko, E.; Kaposloz, H., Myronets, S., Zazymko, O., \& Osodlo, V. (2021). Influence of Characteristics of Self-actualization and Coping Behavior on Resistance of Teachers to Professional Stressors and Emotional Burnout. International Journal of Organizational Leadership, 10(1), 1-14. https://doi.org/10.33844/ijol.2021.60514 